\title{
$\alpha 2$-Adrenoceptor Stimulation Transforms Immune Responses in Neuritis and Blocks Neuritis-Induced Pain
}

\author{
E. Alfonso Romero-Sandoval, ${ }^{1}$ Charles $\mathrm{McCall}^{2}{ }^{2}$ and James C. Eisenach ${ }^{1}$ \\ ${ }^{1}$ Department of Anesthesiology and Center for the Study of Pharmacologic Plasticity in the Presence of Pain, and ${ }^{2}$ Department of Internal Medicine, Wake \\ Forest University School of Medicine, Winston-Salem, North Carolina 27157-1009
}

\begin{abstract}
Neuropathic pain may be primarily driven by immune responses in peripheral nerves. Peripherally released catecholamines may exacerbate neuropathic pain and also modulate immune responses in a complex and sometimes opposing manner by actions on multiple adrenoceptor subtypes. We showed previously that injection of the $\alpha 2$-adrenoceptor agonist clonidine at the site of peripheral nerve injury reduces pain behavior and local tissue pro-inflammatory cytokine content in rats. The current study used a model of acute inflammatory neuritis to test the efficacy and mechanisms of action of $\alpha 2$-adrenoceptor stimulation to reduce pain. Zymosan, injected on the sciatic nerve, caused hypersensitivity to mechanical stimuli ipsilateral to injection and contralaterally, so-called mirror image pain. Ipsilateral hypersensitivity was inhibited dose-dependently by perineural injection of clonidine. Zymosan increased leukocyte number at the site of injection $3 \mathrm{~d}$ later as well as their content of interleukin $1 \alpha$ (IL-1 $\alpha$ ), IL-1 $\beta$, and IL-6. Perineural clonidine prevented both the increase in leukocyte number and cytokine expression induced by zymosan. Additionally, clonidine reduced the capacity of leukocytes to express pro-inflammatory cytokines as assessed by treatment of cells ex vivo with lipopolysaccharide, whereas no repression of IL-10 production occurred. Clonidine reduced the number of macrophages and lymphocytes as well as their expression of tumor necrosis factor $\alpha$. All of the effects of clonidine were prevented by coadministration of an $\alpha 2 \mathrm{~A}$-adrenoceptor-preferring antagonist. These results suggest that $\alpha 2$-adrenoceptor stimulation transforms cytokine gene expression, especially in macrophages and lymphocytes from a pro- to an anti-inflammatory profile in the setting of neuritis, likely relieving neuritis-induced pain by this mechanism.
\end{abstract}

Key words: clonidine; neuritis; cytokines; $\alpha 2$-adrenoceptors; neuropathic pain; inflammation

\section{Introduction}

Neural immune interactions likely contribute to the generation and maintenance of chronic pain after nerve injury. In the periphery, pro-inflammatory cytokines, especially interleukin $1 \beta$ (IL-1 $\beta$ ) and tumor necrosis factor $\alpha$ (TNF $\alpha$ ), exert direct excitatory effects on sensory afferents (Ferreira et al., 1988; Cunha et al., 1992; Sorkin et al., 1997) and are transported centrally where they further sensitize pain transmission (Shubayev and Myers, 2002). In the spinal cord, microglia become activated after peripheral nerve injury, induce central sensitization (DeLeo and Yezierski, 2001), and may underlie extension of pain to the contralateral side, so-called mirror image pain (Twining et al., 2004).

Sympathetic nervous system activity likely exacerbates some neuropathic pain states (Koltzenburg and McMahon, 1991), considered to reflect novel expression of excitatory $\alpha$-adrenoceptors on nociceptors (Sato and Perl, 1991) and sprouting of sympathetic fibers to surround sensory afferent terminals and cell bodies (McLachlan et al., 1993). Sympathetic ner-

\footnotetext{
Received July 20, 2005; revised Aug. 17, 2005; accepted Aug. 23, 2005.

This work was supported in part by National Institutes of Health Grants GM35523 and NS41386. We thank Julie Wieseler Frank for help with experimental details.

Correspondence should be addressed to Dr. James C. Eisenach, Department of Anesthesiology and Center for the Study of Pharmacologic Plasticity in the Presence of Pain, Wake Forest University School of Medicine, Medical Center Boulevard, Winston-Salem, NC 27157-1009. E-mail: jim@eisenach.us.

DOI:10.1523/JNEUROSCI.2995-05.2005

Copyright $\odot 2005$ Society for Neuroscience $\quad 0270-6474 / 05 / 258988-07 \$ 15.00 / 0$
}

vous system effects on immune cells in pain states have been mostly ignored, although many classes of leukocytes express adrenoceptors (Josefsson et al., 1996) and leukocyte responses to challenge can be modulated by increasing sympathetic nervous system activity or by exogenous adrenoceptor agonists (Moynihan et al., 2004).

The $\alpha 2$-adrenoceptor agonist clonidine reduces hypersensitivity in animals with peripheral nerve injury (Yaksh et al., 1995) and provides analgesia in neuropathic pain patients after spinal injection (Eisenach et al., 1995). The effect of clonidine by this route occurs rapidly and lasts for a few hours. In contrast, when clonidine is injected at the site of peripheral nerve injury, it also reduces hypersensitivity, but with an onset of days and duration of $>1$ week (Lavand'homme et al., 2002). This slow time course may reflect clonidine-induced changes in recruitment and function of immune cells at the site of inflammation, as supported by the observation that perineural clonidine reduces proinflammatory cytokine content in the injured peripheral nerve when administered at the time of injury (Lavand'homme and Eisenach, 2003). This effect of clonidine is unexpected, because a report indicated that $\alpha 2$-adrenoceptor stimulation increases TNF $\alpha$ production by lipopolysaccharide (LPS)-challenged macrophages (Spengler et al., 1990), which would promote rather than reduce inflammation.

To further understand the regulation of immune responses to nerve injury by $\alpha$-adrenoceptors, the current study used a model 
of acute inflammatory neuritis from zymosan injection on the sciatic nerve (Chacur et al., 2001). Zymosan causes hypersensitivity to mechanical stimulation of the hindpaw, which is dependent on expression of IL-1, IL-6, TNF $\alpha$, and reactive oxygen species and complement at the site of inflammation (Twining et al., 2004) and which is also dependent on activated microglia in the spinal cord (Milligan et al., 2003). Because lymphocytes (Titnchi and Clark, 1984) and macrophages (Spengler et al., 1990) express $\alpha 2$-adrenoceptors, we hypothesized that the phenotype of these leukocytes would be altered by clonidine and thereby repress hypersensitivity. To test this, we determined whether $\alpha 2$ adrenoceptor stimulation would reduce hyperalgesia in acute inflammatory neuritis and, if so, assessed the cell type(s) and cytokine gene expression changes by which these receptors act.

\section{Materials and Methods}

Animals and surgery. Male Wistar rats, weighing 200-300 g on the day of surgery, were anesthetized, and a catheter was implanted in the left hindlimb as described previously (Chacur et al., 2001). The catheter consisted of a single distal port SILASTIC tubing attached to a piece of gelfoam that was wrapped loosely around the sciatic nerve at mid-thigh level and anchored to adjacent muscles with 3-0 silk suture. The external end of the catheter was passed subcutaneously to the midline just rostral to the tail base, where it was protected by means of a plastic and aluminum frame and sealed with rubber and silicon glue, as described previously (Milligan et al., 1999). The catheter location was verified at the end of the experiment, and only animals with correctly placed catheters were included in data analysis.

Drugs and treatments. Drugs were administered in the conscious animal through the perisciatic nerve catheter $4-5 \mathrm{~d}$ after implantation. The drugs used were zymosan in incomplete Freund's adjuvant (IFA) as vehicle (both from Sigma, St. Louis, MO), clonidine in saline (Roxane Laboratories, Columbus, $\mathrm{OH})$, and the $\alpha 2 \mathrm{~A}$-adrenoceptor-preferring antagonist (+)-2-((4,5-dihydro-1H-imidazol-2-yl)methyl)-2,3-dihydro1-methyl-1H-isoindole (BRL44408) in saline (Tocris, Ellisville, MO).

Animals received a single dose of zymosan $(40 \mu \mathrm{g}$ in $50 \mu \mathrm{l})$ or an equivalent volume of IFA. This zymosan dose produces hypersensitivity to mechanical stimulation ipsilateral to injection as well as mirror image hypersensitivity contralaterally (Chacur et al., 2001). Three hours after zymosan or vehicle injection, animals received a perisciatic nerve catheter injection of saline, clonidine $(10,20$, or $30 \mu \mathrm{g})$, or BRL44408 $(30 \mu \mathrm{g})$, alone or with clonidine $(30 \mu \mathrm{g})$, in a total injection volume of $60 \mu \mathrm{l}$.

Behavioral testing. The withdrawal threshold was measured twice at 10 min intervals ipsilaterally and contralaterally to drug injection using calibrated von Frey filaments (Stoelting, Wood Dale, IL) and an up-down statistical method (Chaplan et al., 1994), and the average of these values was used for data analysis. The withdrawal threshold was determined in each animal before catheter implantation, $4-5 \mathrm{~d}$ after catheter implantation (immediately before zymosan or vehicle injection), $3 \mathrm{~h}$ after zymosan or vehicle injection (immediately before clonidine, BRL44408, or saline injection), and $1-3 \mathrm{~d}$ thereafter. The groups were IFA plus saline $(n=11)$, IFA plus clonidine $(n=10)$, zymosan plus saline $(n=15)$, zymosan plus clonidine $(10,20$, or $30 \mu \mathrm{g} ; n=6,5$, and 13 , respectively), and zymosan plus BR44408 plus clonidine $(n=10)$. The investigator was blinded to drug treatment in all experiments.

Withdrawal thresholds were converted to percentage of maximum possible effect according to the following formula: (withdrawal threshold after drug - withdrawal threshold $3 \mathrm{~h}$ after zymosan injection) $\times 100 /$ (withdrawal threshold before zymosan injection - withdrawal threshold $3 \mathrm{~h}$ after zymosan injection).

Leukocyte preparation and quantification. Rats were anesthetized with halothane and decapitated immediately after the last behavioral test. The perisciatic nerve gelfoam was dissected and mechanically dissociated in HBSS $(20 \mathrm{ml})$ by means of micro-forceps in a plastic Petri dish, as described previously (Gazda et al., 2001). Only preparations in which all the gelfoam could be retrieved were used. The suspension was filtered using a $70 \mu \mathrm{m}$ nylon mesh to separate the leukocytes from the gelfoam. Total leukocyte number per gelfoam insert was determined manually using a hemocytometer, and cell viability was determined by trypan blue exclusion, using $25 \mu \mathrm{l}$ of $0.4 \%$ trypan blue solution (Life Technologies, Grand Island, NY) mixed with $25 \mu \mathrm{l}$ of cell suspension. Leukocyte suspensions were concentrated to $10^{7}$ cells $/ \mathrm{ml}$ in HBSS, and aliquots of $200 \mu$ l were frozen for subsequent measurement of cytokine content. The group sizes were IFA plus saline $(n=9)$, IFA plus clonidine $(n=7)$, zymosan plus saline $(n=11)$, zymosan plus clonidine $(30 \mu \mathrm{g} ; n=11)$, and zymosan plus BR44408 plus clonidine $(n=9)$.

Cytokine measurement. Aliquots were thawed, sonicated for $10 \mathrm{~s}$, and centrifuged at $1500 \times \mathrm{g}$ for $10 \mathrm{~min}$ at $4^{\circ} \mathrm{C}$. Two samples of $50 \mu \mathrm{l}$ of each supernatant were used immediately for cytokine measurement, and the results were averaged for subsequent data analysis. A nine-plex, beadbased rat cytokine immunoassay kit (Bio-Rad, Hercules, CA) was used for simultaneous detection of granulocyte macrophage-colonystimulating factor (GM-CSF), interferon $\gamma$ (IFN $\gamma$ ), IL - 10, IL- $1 \alpha$, IL- $1 \beta$, IL-2, IL-4, IL-6, and TNF $\alpha$ concentrations, following the manufacturer's instructions and as validated previously (Hulse et al., 2005). Multiwavelength fluorescence and cytokine concentrations were determined with a luminometer (Luminex 100 system; Luminex, Austin, TX) and Bio-Rad software. Concentrations of cytokines in samples were within the linear range of the assay in all cases. Cytokine content in total leukocyte preparations was determined for IFA plus saline $(n=8)$, zymosan plus saline $(n=9)$, zymosan plus clonidine ( $30 \mu \mathrm{g} ; n=9)$, and zymosan plus BR44408 plus clonidine $(n=8)$. Values are expressed as nanograms of cytokine $/ 10^{6}$ leukocytes.

Leukocyte subtype analysis and TNF $\alpha$ expression. Leukocyte subtypes and their TNF $\alpha$ expression were analyzed by flow cytometry using FACScan (Becton Dickinson, Franklin Lakes, NJ) and Cell Quest software (Becton Dickinson). One million cells were incubated for $30 \mathrm{~min}$ with surface monoclonal antibodies (mAbs), followed by cell fixation and permeabilization and $30 \mathrm{~min}$ of incubation with intracellular mAbs. Cells were kept in $1 \%$ paraformaldehyde overnight until flow cytometry was performed. To characterize leukocyte populations, we first identified hematopoietic cells using a mouse anti-rat CD45 mAb conjugated with CyChrome $\left(1 \mu \mathrm{g} / 10^{6}\right.$ cells; BD Biosciences, San Jose, CA $)$ as described previously (Brack et al., 2004). Only presumed viable cells were assessed, using standard forward- and side-scatter analysis. To identify cell type, the cell suspension was incubated with CD45 mAb plus a second $\mathrm{mAb}$ conjugated with R-phycoerythrin as follows: mouse anti-rat ED1 for monocytes/macrophages (Brack et al., 2004), mouse anti-rat CD8 for cytotoxic T-lymphocytes (Ikezumi et al., 2004), mouse anti-rat CD4 for helper T-lymphocytes (Katz et al., 1990), mouse anti-rat CD161 for natural killer cells (Durante-Mangoni et al., 2004), mouse anti-rat OX-62 for dendritic cells (Yan et al., 2004), or mouse anti-rat RP-1 for granulocytes (Brack et al., 2004). All mAbs were from Serotec (Raleigh, NC) and used in a volume of $10 \mu \mathrm{l} / 10^{6}$ cells, except for the mAb for RP-1, which was obtained from BD Biosciences and used in a concentration of 1 $\mu \mathrm{g} / 10^{6}$ cells. To assess the proportion of each cell type expressing TNF $\alpha$, cells were incubated with fluorescein isothiocyanate anti-mouse/rat TNF $\alpha\left(1.5 \mu \mathrm{g} / 10^{6}\right.$ cells; eBioscience, San Diego, CA $)$ as characterized previously (Sheehan et al., 1989). For intracellular staining (ED1 and $\mathrm{TNF} \alpha$ ), cells were fixed and permeabilized with medium (Leucoperm; Serotec) following the instructions of the manufacturer. The specificity of the staining was verified by incubation with appropriate isotypematched control antibodies. Leukocyte subtype analysis was determined for IFA plus saline $(n=7)$, zymosan plus saline $(n=8)$, and zymosan plus clonidine ( $30 \mu \mathrm{g} ; n=7)$.

In vitro challenge. Leukocytes retrieved from the gelfoam of animals treated in vivo $3 \mathrm{~d}$ earlier with zymosan plus saline or zymosan plus clonidine were challenged in vitro with LPS (Sigma) or saline. A final concentration of $10^{6}$ cells $/ \mathrm{ml}$ was prepared in RPMI medium (RPMI $1640,10 \%$ fetal bovine serum, $1 \%$ penicillin, $1 \%$ streptomycin, and $1 \%$ L-glutamine) and treated with LPS ( $100 \mathrm{ng} / \mathrm{ml}$ final concentration) or saline immediately after preparation of the leukocytes. Cells were incubated overnight $(16-18 \mathrm{~h})$ at $37^{\circ} \mathrm{C}$ in $95 \% \mathrm{O}_{2} / 5 \% \mathrm{CO}_{2}$. Supernatants were frozen at $-80^{\circ} \mathrm{C}$ until cytokines were measured using the nine-plex, bead-based immunoassay described above.

Statistical analysis. Data are presented as mean \pm SEM. The effects of 


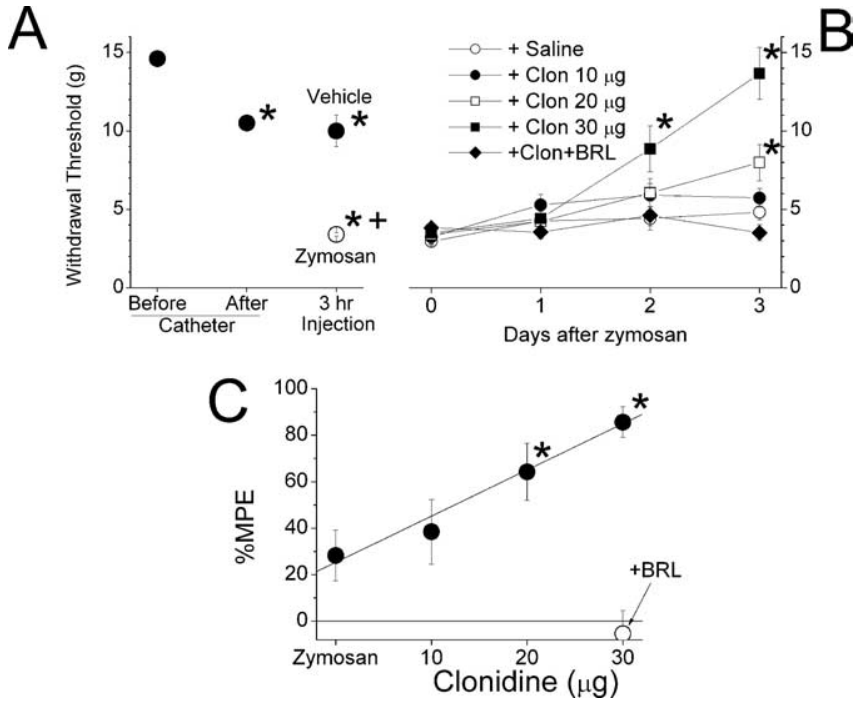

Figure 1. Withdrawal threshold ipsilateral to perineural injections. $\boldsymbol{A}$, The withdrawal threshold to von Frey stimulation ipsilateral to perineural catheterization before and after catheter implantation and $3 \mathrm{~h}$ after zymosan or vehicle. ${ }^{*} p<0.05$ compared with before the catheter value; ${ }^{+} p<0.05$ compared with vehicle. $\boldsymbol{B}$, The withdrawal threshold to von Frey stimulation ipsilateral to perineural injection of zymosan on day 0 , followed in $3 \mathrm{~h}$ by saline or different doses of clonidine alone or $30 \mu \mathrm{g}$ of clonidine plus $30 \mu \mathrm{g}$ of the $\alpha 2$-adrenoceptor antagonist BRL44408. ${ }^{*} p<0.05$ compared with zymosan plus saline; ${ }^{+} p<0.05$ compared with zymosan plus $30 \mu \mathrm{g}$ of clonidine. $C$, The percentage of maximum possible effect (MPE) to return withdrawal threshold to pre-zymosan treatment values $3 \mathrm{~d}$ after perineural injection of zymosan alone or zymosan followed by clonidine $(10,20$, or $30 \mu \mathrm{g}) .{ }^{*} p<0.05$ compared with zymosan alone. Each value represents the mean \pm SE of 6-15 animals. Zym, Zymosan; sal, saline; clo, clonidine; clo10, clo20, and clo30, 10, 20, and $30 \mu \mathrm{g}$ of clonidine, respectively; BRL, BRL44408.

catheter implantation or zymosan or IFA injections on the withdrawal threshold were determined using a repeated-measures, two-way ANOVA, followed by Dunnett's test. Comparisons among groups for cytokine concentrations, leukocyte subtype, and the proportion of each expressing TNF $\alpha$ were performed using $t$ tests, Mann-Whitney $U$ tests, one-way ANOVA followed by the Student-Newman-Keuls test, or the Kruskal-Wallis test as appropriate. $p<0.05$ was considered significant.

\section{Results}

The effect of clonidine on zymosan-induced hypersensitivity Implantation of the perisciatic catheter and gelfoam resulted in a slightly but significantly reduced withdrawal threshold ipsilateral to surgery (Fig. $1 A$ ). In control experiments, IFA alone failed to further reduce withdrawal threshold ipsilateral to injection. In contrast, zymosan significantly reduced the withdrawal threshold $3 \mathrm{~h}$ later, with no recovery over a $3 \mathrm{~d}$ period (Fig. $1 A, B$ ). Clonidine dose-dependently reversed zymosan-induced hypersensitivity ipsilateral to injection, with an onset of $\geq 48 \mathrm{~h}$ after injection (Fig. 1B). Resolution of hypersensitivity beyond $3 \mathrm{~d}$ from zymosan injection in control animals precluded study beyond this period (data not shown). However, the clonidine inhibition of hypersensitivity $3 \mathrm{~d}$ after injection was dose dependent with a threshold of $20 \mu \mathrm{g}$ and a linear dose-response (Fig. $1 B, C$ ). Coadministration of the $\alpha 2 \mathrm{~A}$-preferring antagonist BRL44408 prevented the reversal of clonidine on zymosan-induced hypersensitivity (Fig. $1 B, C$ ).

Perineural zymosan injection also reduced the withdrawal threshold on the contralateral hindpaw (Fig. 2), as described previously (Twining et al., 2004). Perineural clonidine failed to alter contralateral hypersensitivity (Fig. 2), in stark contrast to its efficacy ipsilateral to zymosan injection.

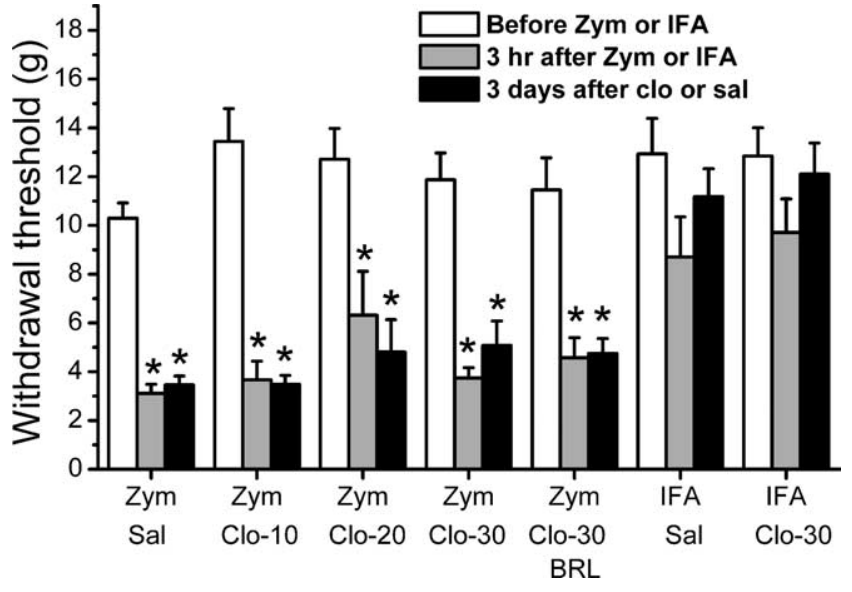

Figure 2. Withdrawal threshold contralateral to perineural injections. The withdrawal threshold to von Frey stimulation contralateral to perineural catheterization before and $3 \mathrm{~h}$ after zymosan (Zym) or IFA injection and then $3 \mathrm{~d}$ after perineural injection of saline (sal) or clonidine (clo; 10,20 , or $30 \mu \mathrm{g}$ alone) or with the $\alpha 2 \mathrm{~A}$-adrenoceptor antagonist BRL44408 (BRL) is shown. Each value represents the mean $+\mathrm{SE}$ of $6-15$ animals. ${ }^{*} p<0.05$ compared with before zymosan or IFA injection. Clo-10, Clo-20, and Clo-30, 10, 20, and $30 \mu \mathrm{g}$ of clonidine, respectively.

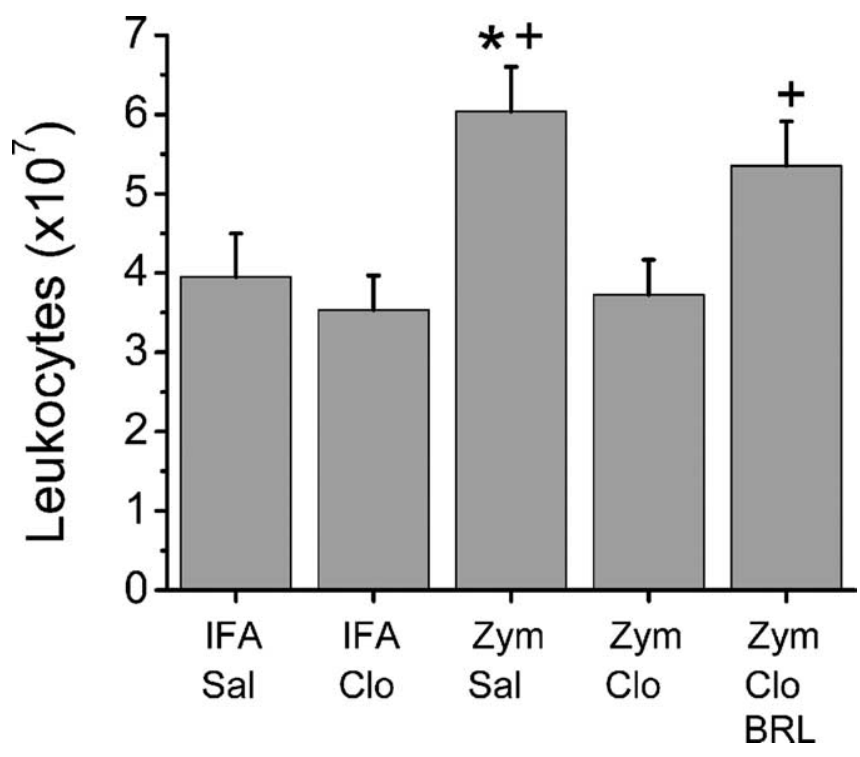

Figure 3. Leukocyte number. The number of leukocytes retrieved from gelfoam $3 \mathrm{~d}$ after perineural injection of IFA or zymosan (Zym), followed by saline (Sal) or clonidine (Clo; $30 \mu \mathrm{g})$, alone or with BRL44408 (BRL), is shown. Each bar represents the mean + SE of 7-11 animals. ${ }^{*} p<0.05$ compared with IFA plus saline; ${ }^{+} p<0.05$ compared with zymosan plus $30 \mu \mathrm{g}$ of clonidine.

\section{Total leukocyte number and their cytokine expression}

More than $95 \%$ of cells prepared from gelfoam in all groups excluded trypan blue and were considered viable. The leukocyte number increased 1.5 fold $3 \mathrm{~d}$ after perineural injection of zymosan compared with saline control (Fig. 3). Perineural clonidine had no effect on leukocyte number in animals treated previously with saline but completely prevented zymosan-induced leukocytosis (Fig. 3). This effect of clonidine was prevented by coadministration of BRL44408 (Fig. 3).

In addition to the effects on leukocyte number, zymosan and clonidine also altered cytokine content of the leukocytes themselves. As such, there was a generalized pattern of increased cytokine expression in leukocytes $3 \mathrm{~d}$ after perineural injection of 

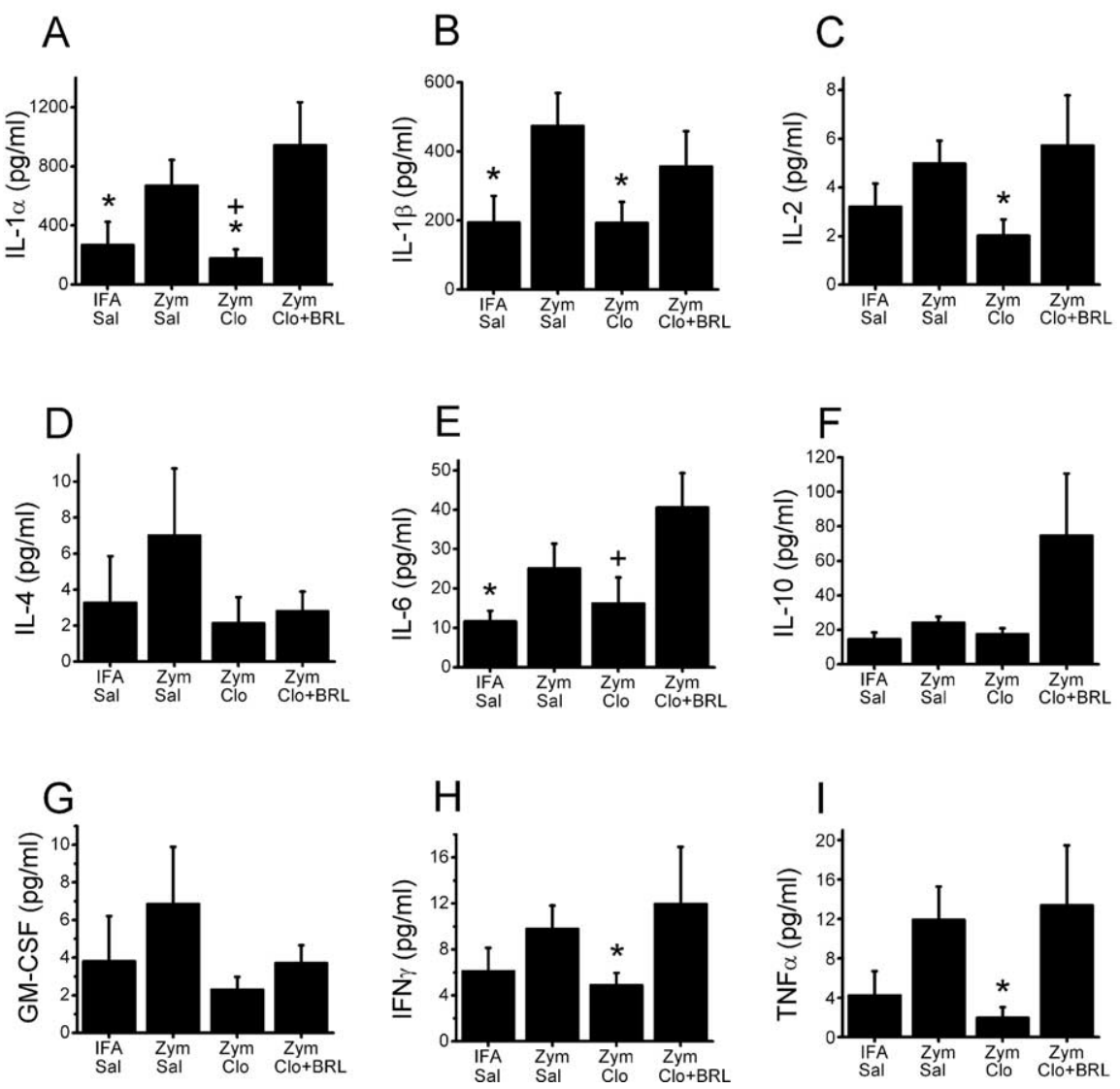

Figure 4. Leukocyte cytokine expression. Cytokine content, expressed as picograms per milliliter in suspensions of $10^{7}$ cells $/ \mathrm{ml}$ of leukocytes retrieved from gelfoam $3 \mathrm{~d}$ after perineural injection of IFA or zymosan (Zym), followed by saline (Sal) or clonidine $(\mathrm{Cl} 0 ; 30 \mu \mathrm{g})$ alone or with BRL44408 (BRL) is shown. $\boldsymbol{A}$, IL-1 $\alpha ; \boldsymbol{B}$, IL-1 $\beta ; \boldsymbol{C}$, IL-2; D, IL-4; $\boldsymbol{E}$, IL-6; $\boldsymbol{F}$, IL-10; $\boldsymbol{G}, \mathrm{GM}-\mathrm{CSF} ; \boldsymbol{H}$, IFN $\gamma ; \boldsymbol{I}$, TNF $\alpha$. Each bar represents the mean + SE of eight to nine animals. ${ }^{*} p<0.05$ compared with zymosan plus saline; ${ }^{+} p<0.05$ compared with zymosan plus clonidine and BRL.

\section{Leukocyte subtype analysis and TNF $\alpha$ expression}

The 1.5-fold increase in total leukocytes in gelfoam $3 \mathrm{~d}$ after perineural zymosan injection was attributable to a twofold to threefold increase in monocytes/macrophages and cytotoxic and helper T-lymphocytes and a nonsignificant 30\% increase in the number of granulocytes (Fig. 5A). Perineural clonidine did not affect the number of granulocytes after zymosan-induced neuritis and completely blocked the twofold to threefold increase in monocytes/macrophages as well as helper and cytotoxic T-lymphocytes (Fig. $5 A)$.

Zymosan treatment produced a general increase in the proportion of leukocytes expressing TNF $\alpha$, with statistically significant increases in granulocyte and helper T-cell subtypes (Fig. 5B). Clonidine treatment generally reduced TNF $\alpha$ expression, with statistically significant reductions compared with zymosan plus saline in monocytes/macrophages and cytotoxic and helper T-cells (Fig. 5B).

\section{In vitro challenge}

Cells harvested from zymosan plus salinetreated animals responded to the LPS challenge ex vivo with significantly increased production of the pro-inflammatory cytokines IL- $1 \alpha$, IL- $1 \beta$, IL-2, IL-6, GM-CSF, and TNF $\alpha$ (Fig. 6). For example, TNF $\alpha$ production increased 2.8-fold to the LPS challenge in cells from zymosan plus saline-treated animals but only 1.7 -fold to

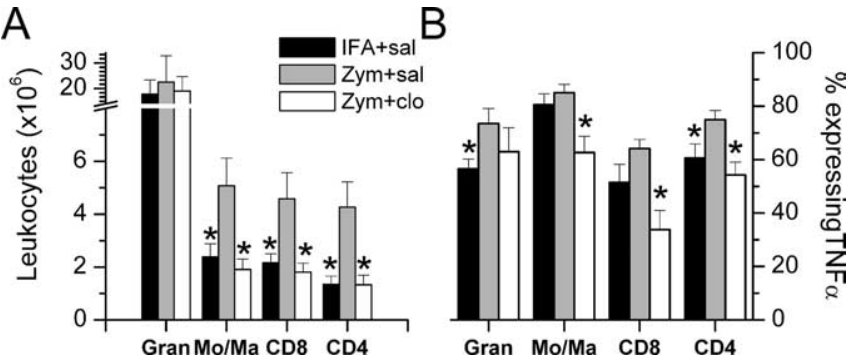

Figure 5. The number of leukocyte subsets and their TNF $\alpha$ expression. $\boldsymbol{A}$, The number of leukocytes per gelfoam isolate identified by flow cytometry to be granulocytes (Gran), monocytes/macrophages (Mo/Ma), CD8 (cytotoxic) T-lymphocytes, and CD4 (helper) T-lymphocytes in animals treated in vivo $3 \mathrm{~d}$ earlier with perineural IFA or zymosan (Zym), followed by saline (sal) or $30 \mu \mathrm{g}$ of clonidine (clo). B. Proportion of leukocytes expressing TNF $\alpha$ of the same subsets. Each bar represents the mean $+\mathrm{SE}$ of seven to eight animals. ${ }^{*} p<0.05$ compared with zymosan plus saline.

zymosan compared with saline control, with statistically significant increases in pro-inflammatory IL- $1 \alpha$, IL- $1 \beta$, and IL-6 (Fig. 4). Compared with zymosan plus saline, perineural clonidine reduced cytokine expression in leukocytes to a broad extent, with statistically significant reductions in IL- $1 \alpha$, IL- $1 \beta$, IL-2, IFN $\gamma$, and TNF $\alpha$ (Fig. 4). The effect of clonidine in each case was inhibited by coadministration of BRL44408 (Fig. 4). the same challenge in cells that had been exposed to clonidine in vivo. In contrast, cells harvested from zymosan plus clonidinetreated animals responded to the LPS challenge with a significantly induced production of these pro-inflammatory cytokines (Fig. 6I). Additionally, the LPS challenge failed to increase production of the anti-inflammatory cytokine IL-10 in zymosan plus saline-treated animals but significantly increased production of this cytokine in cells from clonidine-treated animals (Fig. 6F). A similar trend occurred with the anti-inflammatory cytokine IL-4 (Fig. 6D).

\section{Discussion}

This study shows the following: (1) zymosan treatment of the sciatic model of neurogenic pain increases hypersensitivity both ipsilaterally and contralaterally; (2) this altered physiological phenotype correlates with an increase in the total leukocyte count assessed in gelfoam; (3) constitutive increases in inflammatory cytokines parallel the increase in leukocyte number; (4) locally administered clonidine by receptor-specific mechanisms abrogates ipsilateral (but not contralateral) hypersensitivity in parallel with reductions in pro-inflammatory mediators with no reduction or apparent increases in anti-inflammatory mediators, further favoring repression of inflammation; (5) clonidine also limits the capacity of exudative leukocytes to express pro-inflammatory genes without concomitant repression of anti-inflammatory products; (6) the effect of clonidine may be cell type specific (macrophages and 
T-lymphocytes); and (7) contralateral hypersensitivity induced by zymosan produces pain by a mechanism that is not entirely dependent on pro-inflammatory cytokine content of leukocytes surrounding the ipsilateral sciatic nerve. Together, these unexpected results clarify the actions of catecholamines on immune responses and provide the rationale for new approaches to the treatment of neuropathic pain.

\section{Immune response to}

\section{perineural zymosan}

Three days after zymosan injection, the number of leukocytes in the perineural environment increased, as did their activation state, evidenced by increased IL-1 and IL-6 expression. It may appear paradoxical that TNF $\alpha$, which drives upregulation of IL- 1 and -6 , is not increased by zymosan. It is possible that $\mathrm{TNF} \alpha$ immunoreactivity, measured in the FACS analysis, missed an increase in active cytokine among total immunoreactivity, including proforms, or that the variability in $\mathrm{TNF} \alpha$ precluded demonstration of significance from the strong trend $(p=0.07)$ of increased TNF $\alpha$. Cytokine expression has not been quantified previously $3 \mathrm{~d}$ after zymosan injection in this neuritis model, although others have shown increased TNF $\alpha$ and IL- $1 \beta$ secretion ex vivo from leukocytes recovered from gelfoam at $24 \mathrm{~h}$ after perineural zymosan injection (Gazda et al., 2001). Zymosan activates nuclear factor $\kappa \mathrm{B}$ by binding to Toll-like receptor 2 (TLR-2) and TLR-6 (Underhill et al., 1999; Young et al., 2001) and likely contributes to pro-inflammatory cytokine gene expression by this mechanism. These cytokines play key roles in the generation of zymosaninduced hypersensitivity, because perisciatic nerve injection of antibodies or receptor antagonists to these cytokines prevents the development of ipsilateral and bilateral hypersensitivity (Twining et al., 2004). Additionally, haplotypes with enhanced IL-6 signaling impart a more than fivefold increased risk in humans for sciatica and pain associated with intervertebral disc disease (Noponen-Hietala et al., 2005), consistent with relevance of this cytokine to clinical pain.

Although the number of perineural leukocytes is not increased 3 and $24 \mathrm{~h}$ after zymosan (Gazda et al. 2001), we demonstrate a leukocytosis $3 \mathrm{~d}$ after zymosan, probably reflecting the concomitant release of chemokines and cytokines. The population makeup of leukocytes in the gelfoam from control animals typifies a foreign body-induced exudate, and zymosan also increases leukocyte number primarily by increasing T-lymphocytes and monocytes/macrophages. Thus, zymosan increases both the total number of leukocytes and their cellular expression of proinflammatory cytokines in this foreign body, representing a large increase in cytokine content in the environment surrounding the nerve. The parallel time course between the number of proinflammatory leukocytes and hypersensitivity after nerve injury (Sommer and Schafers, 1998; Shamash et al., 2002; Moalem et al., 2004) also supports the importance of zymosan-induced leukocytosis and immune activation to persistent pain in this model.
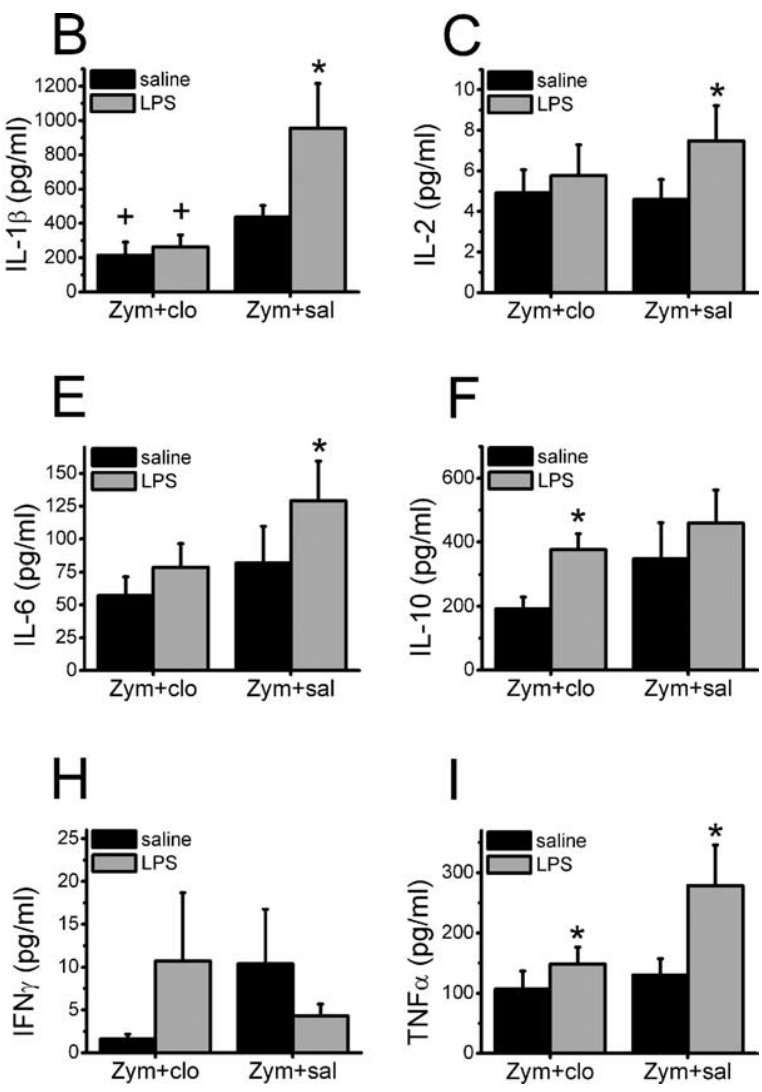

Figure 6. Cytokine expression from the LPS challenge. Cytokine concentration after incubation in vitro with saline or LPS in the $\mu \mathrm{g}$ of clonidine (clo). Each bar represents the mean + SE of six experiments. $\boldsymbol{A}, \mathrm{IL}-1 \alpha ; \boldsymbol{B}, \mathrm{IL}-1 \beta ; \boldsymbol{C}, \mathrm{IL}-2 ; \boldsymbol{D}, \mathrm{IL}-4 ; \boldsymbol{E}, \mathrm{IL}-6 ; \boldsymbol{F}, \mathrm{IL}-10 ;$ $\mathbf{G}, \mathrm{GM}-\mathrm{CSF} ; \boldsymbol{H}$, IFN $\gamma ; \boldsymbol{I}, \mathrm{TNF} \alpha .{ }^{*} p<0.05$ compared with in vitro saline control; ${ }^{+} p<0.05$ compared with in vivo zymosan plus

We recognize that intracellular cytokine content, as measured by immunoreactivity in cell homogenates or by FACS in the current study, does not measure released cytokine concentrations, and it is conceivable that cytokine release may differ from expression.

\section{$\alpha 2$-Adrenoceptors transform the immune response to acute neuritis}

Perineural clonidine prevented both the zymosan-induced increase in T-lymphocytes and monocytes/macrophages as well as their expression of pro-inflammatory cytokines. The proinflammatory cytokines that were increased after zymosan injection in control animals (IL-1, IL-6, TNF $\alpha$ ) attract immune cells, and their reduced expression by clonidine would thereby prevent leukocytosis. $\alpha 2$-Adrenoceptor stimulation induces apoptosis in lymphocytes (Stevenson et al., 2001), providing an alternative explanation for reduced leukocytosis in clonidine-treated animals. This mechanism seems unlikely, however, because clonidine did not alter leukocyte number in gelfoam in the absence of zymosan or the number of leukocytes that failed to exclude trypan blue.

In contrast to its effect on pro-inflammatory mediators, clonidine failed to repress anti-inflammatory cytokine expression. Because pro-inflammatory cytokines generate hypersensitivity and pain (see above) and because IL-10 reduces hypersensitivity and pain (Milligan et al., 2005), we speculate that clonidine produces analgesia by shifting cytokine expression. The mechanisms by which clonidine affects this change require further study. However, T-lymphocytes and monocytes/macrophages 
constitutively express $\alpha 2$-adrenoceptors (Spengler et al., 1990; Schauenstein et al., 2000) and demonstrate $\alpha 2 \mathrm{~A}$-adrenoceptor immunostaining at the site of nerve injury (Lavand'homme et al., 2002), and therefore likely represent a site of action.

The effects of $\alpha 2$-adrenoceptor activation on macrophages have received little previous attention. In isolated pure preparations of macrophages, $\alpha 2$-adrenoceptor agonists stimulate proinflammatory IL-12 (Kang et al., 2003) and TNF $\alpha$ (Spengler et al., 1990) expression, whereas in a mixed leukocyte preparation from whole-blood, $\alpha 2$-adrenoceptor agonists inhibit LPS-induced TNF $\alpha$ expression (Maes et al., 2000). We propose that $\alpha 2$-adrenoceptor stimulation in the microenvironment of neuritis shifts the macrophage phenotype from pro- to anti-inflammatory. Development of an explant model to mimic this environment would facilitate study of the mechanisms by which $\alpha 2$-adrenoceptors produce this change.

To further test whether clonidine induces a sustained phenotypic change among the leukocytes, we stimulated cells ex vivo with the TLR-4 ligand LPS $3 \mathrm{~d}$ after zymosan injection. LPSinduced increases in production of IL-1, IL-2, IL-6, GM-CSF, and TNF $\alpha$ in cells from zymosan plus saline-treated animals is consistent with a pro-inflammatory profile. Similar responses were obtained with in vitro stimulation by zymosan of leukocytes isolated 3 and $24 \mathrm{~h}$ after in vivo zymosan injection in this model (Gazda et al., 2001). In contrast, clonidine treatment not only reduced pro-inflammatory cytokine expression by leukocytes exposed to zymosan in vivo, it also resulted in a phenotype that later responded to the immunological LPS challenge ex vivo by repression of pro-inflammatory cytokine production with sustained production of the anti-inflammatory cytokine IL-10.

\section{$\alpha$-Adrenoceptors and hypersensitivity from neuritis}

The current study provides one explanation for the analgesic efficacy of peripherally administered clonidine in neuropathic pain. Peripheral nerve injury and resultant Wallerian degeneration stimulates an immunological response throughout the length of the nerve, extending to intact peripheral terminals, resulting in pain and hypersensitivity from exposure of these nerve endings to pro-inflammatory products. Thus, intraplantar cyclooxygenase inhibition relieves hypersensitivity and abnormal excitatory neuropeptide expression in afferents and the spinal cord after nerve injury (Ma and Eisenach, 2003). Although the current study used a model of acute neural inflammation, these parallels in presumed mechanism support inhibition of proinflammatory responses to nerve injury as the basis for efficacy of topical clonidine to treat neuropathic pain and suggest that peripherally restricted $\alpha 2$-adrenoceptor agonists such as oxymetazoline (Afrin), which lack sedative and hypotensive effects, could be applied topically or via perineural injection for analgesia in neuropathic pain.

Perineural clonidine failed to reduce mirror-image pain hypersensitivity in the current study. In contrast, perineural injection of agents that block TNF $\alpha$, IL-6, IL-1, complement activity, or reactive oxygen generation result in a rapid onset repression of bilateral hypersensitivity, lasting for a few hours (Twining et al., 2004). Similarly, acute blockade of microglial function or p38 mitogen-activated protein kinase signaling in the spinal cord immediately after perineural zymosan injection blocks bilateral pain hypersensitivity (Milligan et al., 2003), consistent with a role for activated microglia in contralateral spread of sensitization. Although these studies confirm the relevance of locally produced pro-inflammatory mediators to hypersensitivity, their dramatically different time course compared with clonidine is consistent with acute pharmacological blockade of cytokine action with the former and altered cytokine gene expression by the latter. Additionally, the unilateral blockade of hypersensitivity by clonidine, but not by cytokine blockers, suggests that pro-inflammatory signaling at the site of neuritis is not necessary for the maintenance of contralateral sensitization. Alternatively, proinflammatory cytokine exposure over the $2-3 \mathrm{~d}$ before clonidine reduced hypersensitivity could have been sufficient to make mirror-image pain independent of additional peripheral cytokine-driven signaling to the spinal cord.

In summary, a single perineural injection of clonidine gradually alleviates for days hypersensitivity to mechanical stimulation in a neuritis model of pain via actions on $\alpha 2$-adrenoceptors. Unexpectedly, clonidine transforms the macrophage and T-lymphocyte phenotype in neuritis from pro-inflammatory and pain producing to anti-inflammatory and pain reducing. These results provide the rationale for a novel therapeutic strategy for neuropathic pain and a novel mechanism by which catecholamines regulate immune responses and neural-immune interactions.

\section{References}

Brack A, Rittner HL, Machelska H, Leder K, Mousa SA, Schäfer M, Stein C (2004) Control of inflammatory pain by chemokine-mediated recruitment of opioid-containing polymorphonuclear cells. Pain 112:229-238.

Chacur M, Milligan ED, Gazda LS, Armstrong C, Wang H, Tracey KJ, Maier SF, Watkins LR (2001) A new model of sciatic inflammatory neuritis (SIN): induction of unilateral and bilateral mechanical allodynia following acute unilateral peri-sciatic immune activation in rats. Pain 94:231-244.

Chaplan SR, Bach FW, Pogrel JW, Chung JM, Yaksh TL (1994) Quantitative assessment of tactile allodynia in the rat paw. J Neurosci Methods 53:55-63.

Cunha FQ, Poole S, Lorenzetti BB, Ferreira SH (1992) The pivotal role of tumour necrosis factor alpha in the development of inflammatory hyperalgesia. Br J Pharmacol 107:660-664.

DeLeo JA, Yezierski RP (2001) The role of neuroinflammation and neuroimmune activation in persistent pain. Pain 90:1-6.

Durante-Mangoni E, Wang R, Shaulov A, He Q, Nasser I, Afdhal N, Koziel MJ, Exley MA (2004) Hepatic CD1d expression in hepatitis C virus infection and recognition by resident proinflammatory CD1d-reactive $\mathrm{T}$ cells. J Immunol 173:2159-2166.

Eisenach JC, DuPen S, Dubois M, Miguel R, Allin D, Epidural Clonidine Study Group 1995 Epidural clonidine analgesia for intractable cancer pain. Pain 61:391-399.

Ferreira SH, Lorenzetti BB, Bristow AF, Poole S (1988) Interleukin-1 beta as a potent hyperalgesic agent antagonized by a tripeptide analogue. Nature 334:698-700

Gazda LS, Milligan ED, Hansen MK, Twining CM, Poulos NM, Chacur M, O’Connor KA, Armstrong C, Maier SF, Watkins LR, Myers RR (2001) Sciatic inflammatory neuritis (SIN): behavioral allodynia is paralleled by peri-sciatic proinflammatory cytokine and superoxide production. $\mathrm{J} \mathrm{Pe}$ ripher Nerv Syst 6:111-129.

Hulse RF, Kunkler PE, Fedynyshyn JP, Kraig RP (2005) Optimization of multiplexed bead-based cytokine immunoassays for rat serum and brain tissue. J Neurosci Methods 136:87-98.

Ikezumi Y, Kanno K, Karasawa T, Han GD, Ito Y, Koike H, Toyabe S, Uchiyama M, Shimizu F, Kawachi H (2004) The role of lymphocytes in the experimental progressive glomerulonephritis. Kidney Int 66:1036-1048.

Josefsson E, Bergquist J, Ekman R, Tarkowski A (1996) Catecholamines are synthesized by mouse lymphocytes and regulate function of these cells by induction of apoptosis. Immunology 88:140-146.

Kang BY, Lee SW, Kim TS (2003) Stimulation of interleukin-12 production in mouse macrophages via activation of p38 mitogen-activated protein kinase by alpha2-adrenoceptor agonists. Eur J Pharmacol 467:223-231.

Katz J, Michalek SM, Beagley KW, Eldridge JH (1990) Characterization of rat $\mathrm{T}$ helper cell clones specific for Bacteroides gingivalis antigen. Infect Immun 58:2785-2791.

Koltzenburg M, McMahon SB (1991) The enigmatic role of the sympathetic nervous system in chronic pain. Trends Pharmacol Sci 12:399-402.

Lavand'homme PM, Eisenach JC (2003) Perioperative administration of the $\alpha 2$-adrenoceptor agonist clonidine at the site of nerve injury reduces 
the development of mechanical hypersensitivity and modulates local cytokine expression. Pain 105:247-254.

Lavand'homme PM, Ma W, De Kock M, Eisenach JC (2002) Peri-neural $\alpha_{2 A}$-adrenoceptor activation inhibits spinal cord neuroplasticity and tactile allodynia after nerve injury. Anesthesiology 97:972-980.

Ma W, Eisenach JC (2003) Intraplantar injection of a cyclooxygenase inhibitor ketorolac reduces immunoreactivities of substance $\mathrm{P}$ calcitonin generelated peptide, and dynorphin in the dorsal horn of rats with nerve injury or inflammation. Neuroscience 121:681-690.

Maes M, Lin AH, Kenis G, Egyed B, Bosmans E (2000) The effects of noradrenaline and alpha- 2 adrenoceptor agents on the production of monocytic products. Psychiatry Res 96:245-253.

McLachlan EM, Janig W, Devor M, Michaelis M (1993) Peripheral nerve injury triggers noradrenergic sprouting within dorsal root ganglia. Nature 363:543-546.

Milligan ED, Hinde JL, Mehmert KK, Maier SF, Watkins LR (1999) A method for increasing the viability of the external portion of lumbar catheters placed in the spinal subarachnoid space of rats. J Neurosci Methods 90:81-86.

Milligan ED, Twining C, Chacur M, Biedenkapp J, O’Connor K, Poole S, Tracey K, Martin D, Maier SF, Watkins LR (2003) Spinal glia and proinflammatory cytokines mediate mirror-image neuropathic pain in rats. J Neurosci 23:1026-1040.

Milligan ED, Langer SJ, Sloane EM, He L, Wieseler-Frank J, O'Connor K, Martin D, Forsayeth JR, Maier SF, Johnson K, Chavez RA, Leinwand LA, Watkins LR (2005) Controlling pathological pain by adenovirally driven spinal production of the anti-inflammatory cytokine, interleukin10. Eur J Neurosci 21:2136-2148.

Moalem G, Xu K, Yu L (2004) T lymphocytes play a role in neuropathic pain following peripheral nerve injury in rats. Neuroscience 129:767-777.

Moynihan J, Kruszewska B, Madden K, Callahan T (2004) Sympathetic nervous system regulation of immunity. J Neuroimmunol 147:87-90.

Noponen-Hietala N, Virtanen I, Karttunen R, Schwenke S, Jakkula E, Li H, Merikivi R, Barral S, Ott J, Karppinen J, Ala-Kokko L (2005) Genetic variations in IL6 associate with intervertebral disc disease characterized by sciatica. Pain 114:186-194.

Sato J, Perl ER (1991) Adrenergic excitation of cutaneous pain receptors induced by peripheral nerve injury. Science 251:1608-1610.

Schauenstein K, Felsner P, Rinner I, Liebmann PM, Stevenson JR, Westermann J, Haas HS, Cohen RL, Chambers DA (2000) In vivo immunomodulation by peripheral adrenergic and cholinergic agonists/antagonists in rat and mouse models. Ann NY Acad Sci 917:618-627.

Shamash S, Reichert F, Rotshenker S (2002) The cytokine network of Wal- lerian degeneration: tumor necrosis factor- $\alpha$, interleukin- $1 \alpha$, and interleukin-1 $\beta$. J Neurosci 22:3052-3060.

Sheehan KC, Ruddle NH, Schreiber RD (1989) Generation and characterization of hamster monoclonal antibodeis that neutralize murine tumor necrosis factors. J Immunol 142:3884-3893.

Shubayev VI, Myers RR (2002) Anterograde TNF $\alpha$ transport from rat dorsal root ganglion to spinal cord and injured sciatic nerve. Neurosci Lett 320:99-101.

Sommer C, Schafers M (1998) Painful mononeuropathy in C57BL/Wld mice with delayed wallerian degeneration: differential effects of cytokine production and nerve regeneration on thermal and mechanical hypersensitivity. Brain Res 784:154-162.

Sorkin LS, Xiao W-H, Wagner R, Myers RR (1997) Tumour necrosis factoralpha induces ectopic activity in nociceptive primary afferent fibres. Neuroscience 81:255-262.

Spengler RN, Allen RM, Remick DG, Strieter RM, Kunkel SL (1990) Stimulation of alpha-adrenergic receptor augments the production of macrophage-derived tumor necrosis factor. J Immunol 145:1430-1434.

Stevenson JR, Westermann J, Liebmann PM, Hortner M, Rinner I, Felsner P, Wolfler A, Schauenstein K (2001) Prolonged alpha-adrenergic stimulation causes changes in leukocyte distribution and lymphocyte apoptosis in the rat. J Neuroimmunol 120:50-57.

Titnchi S, Clark B (1984) Alpha-2 adrenoceptors in human lymphocytes: direct characterisation by $\left[{ }^{3} \mathrm{H}\right]$-yohimbine binding. Biochem Biophys Res Commun 121:1-7.

Twining CM, Sloane EM, Milligan ED, Chacur M, Martin D, Poole S, Marsh H, Maier SF, Watkins LR (2004) Peri-sciatic proinflammatory cytokines, reactive oxygen species, and complement induce mirror-image neuropathic pain in rats. Pain 110:299-309.

Underhill DM, Ozinsky A, Hajjar AM, Stevens A, Wilson CB, Bassetti M, Aderem A (1999) The Toll-like receptor 2 is recruited to macrophage phagosomes and discriminates between pathogens. Nature 401:811-815.

Yaksh TL, Pogrel JW, Lee YW, Chaplan SR (1995) Reversal of nerve ligation-induced allodynia by spinal alpha-2 adrenoceptor agonists. J Pharmacol Exp Ther 272:207-214.

Yan H, Miyagi T, Satoh E, Sugiura W, Yamamoto N, Kimura H (2004) Phenotype and function of GM-CSF independent dendritic cells generated by long-term propagation of rat bone marrow cells. Cell Immunol 229:117-129.

Young SH, Ye J, Frazer DG, Shi X, Castranova V (2001) Molecular mechanism of tumor necrosis factor-alpha production in $1 \rightarrow 3$-beta-glucan (zymosan)-activated macrophages. J Biol Chem 276:20781-20787. 